\section{Exposición a agentes de riesgo biológico en trabajadores chilenos. Reporte del Centro de Información Toxicológica de la Pontificia Universidad Católica de Chile (CITUC)}

\author{
PATRICIA CERDA ${ }^{1}$, SANDRA CORTÉS ${ }^{2}$, MARLI BETTINI ${ }^{1,4}$, \\ JUAN JOSÉ MIERES ${ }^{1}$, ENRIQUE PARIS ${ }^{1}$, JUAN CARLOS RÍOS ${ }^{1,3}$
}

\section{Exposure to occupational biological risks. Experience of a toxicology information center}

Background: Exposure to Biological Agents during work is an emergent type of occupational risk. Aim: To characterize occupational biological risk exposure among Chilean workers which have been registered by the Toxicology Information Center, between January 2006 and December 2009. Material and Methods: All incoming calls reporting exposure to biological agents during the studied period were analyzed. The information obtained from the caller was registered using the Communication Record Instrument of the WHO International Programme on Chemical Safety (IPCS INTOX). Results: In the studied period, 77 calls were received. The mean age of exposed patients was $35 \pm 15$ years and $57 \%$ of them were females. The most common involved agents were vaccines for veterinary use (42\%) followed by Loxosceles laeta bites in 16\%. The main routes of exposure were injections, cuts and needle stick injuries in $39 \%$ and stings and bites in 38\%. The highest exposure rates were observed in Southern Chile due to self-inoculation of veterinary vaccines used in the salmon industry (22.7/100.000 actual workers). Fifty-eight percent of calls were from health care workers, and $51 \%$ of them were from health care facilities. Sixty percent of exposures occurred during summer and spring. There was a fourfold higher risk of calls involving women exposed to bites or stings (odds ratio (OR) $4.5\left(\mathrm{CI}_{95}\right.$ 1.5-13.9, $\left.\mathrm{p}<0.01\right)$. Men had a fourfold higher risk of being exposed to vaccines or medications for veterinary use (OR 4.2, $C_{95}$ 1.4-12.6 $\left.p<0.01\right)$. Conclusions: Most calls involving an exposure to a biological agent were caused by self-inoculation of veterinary medications.

(Rev Med Chile 2014; 142: 443-450)

Key words: Epidemiology; Occupational Exposure; Risk; Vaccines.
${ }^{1}$ Centro de Información Toxicológica y de Medicamentos (CITUC). Escuela de Medicina, Pontificia Universidad Católica de Chile. Santiago de Chile.

${ }^{2}$ Departamento de Salud Pública. Escuela de Medicina, Pontificia Universidad Católica de Chile. Santiago de Chile.

${ }^{3}$ Departamento de Laboratorios Clínicos. Escuela de Medicina,

Pontificia Universidad Católica de Chile. Santiago de Chile.

${ }^{4}$ Departamento de salud del niño y del adolescente, Escuela de Enfermería, Pontificia Universidad Católica de Chile. Santiago de Chile.

Los autores declararon no tener conflictos de intereses.

Recibido el 17 de enero de 2013, aceptado el 10 de abril de 2014 .



$\circlearrowleft$ e entiende por riesgo biológico ocupacional la exposición en el lugar de trabajo a agentes vivos capaces de originar cualquier tipo de infección, alergia o toxicidad ${ }^{1}$. Estos agentes abarcan variados microorganismos (virus, bacterias, parásitos, hongos, cultivos celulares, etc.), toxinas $\mathrm{y}$ alergenos que pueden perjudicar la salud. Este riesgo laboral se considera "emergente" ${ }^{2-3}$, debido a que es un "nuevo riesgo", generado por características del trabajo, anteriormente desconocido o menos evidente ${ }^{2}$.

La transmisión ocupacional de enfermedades infecciosas es responsable de un estimado de 320.000 muertes/año a nivel mundial y de 5.000 muertes/año en la Unión Europea ${ }^{4}$. En Chile la información es escasa en cuanto a exposiciones 
ocupacionales y daños a la salud ${ }^{5}$. La información oficial se refiere únicamente a intoxicación por plaguicidas, cuya notificación es obligatoria desde el año $2004^{6}$.

La existencia de riesgo biológico y su conocimiento es relevante en el ambiente laboral debido al contacto con agentes altamente peligrosos, diferentes tipos de exposición, falta de valores límite, presencia de trabajadores con un sistema inmunitario defectuoso y, por lo tanto, más susceptibles al riesgo ${ }^{7}$.

En diversos y numerosos lugares de trabajo los trabajadores pueden exponerse a agentes de riesgo biológico ${ }^{7-8}$. Los grupos de mayor riesgo corresponden a personal de la salud, personal de laboratorios, trabajadores agrícolas, trabajadores en contacto con animales y veterinarios ${ }^{3-9-10}$.

Según la Encuesta Nacional de Empleo, Calidad de Vida, Trabajo y Salud (ENETS) 20092010, aplicada sobre una muestra representativa compuesta por 9.503 trabajadores chilenos $(62,7 \%$ sexo masculino), $12,7 \%$ se expone a agentes de riesgo biológico laboral, lo que corresponde a 1.207 trabajadores $^{11}$.

Si se calcula $12,7 \%$ de la fuerza laboral ocupada a nivel nacional, que corresponde a 7.589.410 trabajadores $^{12}$, se obtiene que 963.855 trabajadores en Chile podrían estar expuestos a agentes de riesgo biológico en su lugar de trabajo.

El objetivo de este estudio fue caracterizar las exposiciones a agentes de riesgo biológico ocupacional en trabajadores chilenos registradas por el Centro de Información Toxicológica y de Medicamentos (CITUC) durante el período comprendido entre el $1^{\circ}$ de enero de 2006 y el 31 de diciembre de 2009.

\section{Material y Método}

Se realizó un estudio descriptivo del total de llamados telefónicos por exposición a agente de riesgo biológico en el lugar de trabajo registrados por el CITUC durante el período comprendido entre el $1^{\circ}$ de enero de 2006 y el 31 de diciembre de 2009. Estos llamados a CITUC se generaron de manera voluntaria. Cada llamado fue atendido por un profesional y registrado en la base de datos del Centro. La información aportada por la persona que realizó el llamado o interlocutor se obtuvo a través de la aplicación del instrumento Commu- nication Record ${ }^{13}$, del International Programme on Chemical Safety (IPCS INTOX) de la Organización Mundial de la Salud (OMS) ${ }^{14}$.

El CITUC funciona $24 \mathrm{~h}$ del día, todos los días del año y tiene cobertura para todo el territorio nacional, por lo tanto, todo llamado que se generó durante el período mencionado fue atendido y registrado con el mismo protocolo, utilizando el mismo instrumento.

Se analizaron las variables: exposición a agente de riesgo biológico laboral, edad por grupo (14-20, 21-39, 40-60, 61-70 años), sexo (femenino-masculino), agente (de acuerdo al criterio de Dutkiewicz et al, $2011^{15}$ y Dutkiewicz et al, $1988^{16}$ ), vía de exposición (inhalatoria, cutánea, digestiva, ocular, inyecciones y lesiones corto-punzantes, picaduramordedura), interlocutor o persona que realiza el llamado a CITUC (trabajador, empleador, personal de salud, compañero de trabajo, familiar), lugar de procedencia del llamado (recinto hospitalario, trabajo, hogar, atención primaria, consulta externa, clínica veterinaria), región (de acuerdo a la nueva distribución político-administrativa del país) y estación del año (otoño, invierno, primavera, verano).

Las variables categóricas se describen según distribución de frecuencias y porcentajes y la variable numérica edad por el promedio y su desviación estándar.

A través de tablas de contingencia se exploró la asociación entre sexo y los agentes de riesgo biológico más relevantes. Se aplicó el estadístico $\chi^{2}$ para comparación de proporciones. Se calcularon Odds Ratios (OR) con sus respectivos intervalos de confianza a $95 \%$ para estimar la magnitud de la asociación entre variables de interés. Se consideró estadísticamente significativo un valor de $\mathrm{p}<0,05$.

Se utilizó información epidemiológica aportada por la ENETS ${ }^{11}$ y por el Instituto Nacional de Estadísticas ${ }^{12}$ para obtener una aproximación de la población en riesgo de exposición a agentes de riesgo biológico ocupacional y para calcular tasas de exposición según región.

\section{Resultados}

Durante el período descrito, el CITUC recibió 77 llamados por exposición a agentes de riesgo biológico en el lugar de trabajo, las que representan $4,3 \%$ del total de exposiciones a tóxicos laborales 
Exposición a agentes de riesgo biológico en trabajadores chilenos - P. Cerda et al

Tabla 1. Distribución de trabajadores expuestos a agentes de riesgo biológico ocupacional según grupo de edad y sexo

\begin{tabular}{|lrrrrrrr|}
\hline $\begin{array}{l}\text { Grupo } \\
\text { de edad } \\
\text { (años) }\end{array}$ & \multicolumn{4}{c}{\begin{tabular}{c}
\multicolumn{2}{c}{ Femenino } \\
\%
\end{tabular}} & \multicolumn{1}{c}{ (n) } & \multicolumn{1}{c}{$\%$} & \multicolumn{3}{c|}{ Total } \\
\hline $14-20$ & 11,7 & $(9)$ & 6,5 & $(5)$ & 18,2 & $(14)$ \\
\hline $21-39$ & 29,9 & $(23)$ & 18,2 & $(14)$ & 48,1 & $(37)$ \\
\hline $40-60$ & 10,3 & $(8)$ & 16,9 & $(13)$ & 27,2 & $(21)$ \\
\hline $61-70$ & 5,2 & $(4)$ & 1,3 & $(1)$ & 6,5 & $(5)$ \\
\hline Total & 57,1 & $(44)$ & 42,9 & $(33)$ & 100,0 & $(77)$ \\
\hline
\end{tabular}

registradas durante el mismo período $(\mathrm{n}=1.774)$, ocupando el tercer lugar después de agentes químicos y de uso industrial $(63,1 \%)$ y plaguicidas y productos de uso agrícola $(31,6 \%)$.

En relación a la distribución según sexo, se observó predominio del sexo femenino $(57,1 \%)$. La edad promedio fue 34,8 años (DE: 14,5$)$ con un rango entre 17 y 70 años. El 48,1\% de los trabajadores tenía edad entre 21 y 39 años (Tabla 1).

Con respecto a la distribución según agente, el principal correspondió a vacunas de uso veterinario $(41,6 \%)$, seguido por araña de rincón $(15,5 \%)$ y picadura de insecto $(10,4 \%)$ (Tabla 2 ).

En relación al agente "vacuna de uso veterinario", el principal porcentaje correspondió a vacuna anti rickettsia salmones (50\%) (Tabla 3).

Las principales vías de exposición correspondieron a inyecciones y lesiones corto-punzantes $(39,0 \%)$, y a picaduras y mordeduras $(37,7 \%)$ (Tabla 4).

Se observó un predominio del sexo femenino para mordeduras y picaduras de arañas, animales e insectos y predominio del sexo masculino para vacunas y medicamentos de uso veterinario (Tabla 5).

Al contrastar el sexo femenino y la picadura o mordedura de arañas, animales e insectos, se observó que el porcentaje de exposición en mujeres fue $59 \%(26 / 44)$, a diferencia de $24,2 \%$ en hombres $(8 / 33)$. Esta diferencia fue estadísticamente significativa $\left(\chi^{2}: 9,2, \mathrm{p}<0,005\right)$ (Tabla $6)$. Existe mayor probabilidad $(4,5)$ de que un llamado recibido por mordedura o picadura se refiera a una trabajadora de sexo femenino (OR: 4,$\left.5 ; \mathrm{IC}_{95 \%}: 1,5-13,9\right)$ con respecto a un trabajador de sexo masculino (Tabla 6).
Tabla 2. Distribución de exposiciones a agente de riesgo biológico ocupacional según agente

\begin{tabular}{|lrr|}
\hline Agente de riesgo biológico & \multicolumn{2}{c|}{ Total } \\
Vacuna veterinaria & \multicolumn{1}{c}{$\%$} & (n) \\
Araña de rincón (Loxosceles laeta) & 41,6 & $(32)$ \\
Insecto & 15,5 & $(12)$ \\
Araña común & 10,4 & $(8)$ \\
Araña del trigo (Latrodectus mactans) & 7,8 & $(6)$ \\
Medicamento de uso veterinario & 5,2 & $(5)$ \\
Alimento/bebida contaminado & 3,9 & $(3)$ \\
Alga & 2,6 & $(2)$ \\
Mordedura de perro & 1,3 & $(1)$ \\
Alacrán & 1,3 & $(1)$ \\
Abeja & 1,3 & $(1)$ \\
\hline Hongo & 1,3 & $(1)$ \\
\hline Planta & 1,3 & $(1)$ \\
\hline Total & 100,0 & $(77)$ \\
\hline
\end{tabular}

Al contrastar el sexo masculino y la exposición a vacunas y medicamentos de uso veterinario, se observó que el porcentaje de exposición en hombres fue $66,6 \%(22 / 33)$, a diferencia de $31,8 \%$ en mujeres (14/44). Esta diferencia fue estadísticamente significativa $\left(\chi^{2}: 9,2, \mathrm{p}<0,005\right)$ (Tabla 7). Existe mayor probabilidad $(4,2)$ de recibir un llamado por exposición a vacunas veterinarias y medicamentos veterinarios en trabajadores de sexo masculino (OR: 4,2, IC95\%: 1,4-12,6) en relación a trabajadoras de sexo femenino (Tabla 7).

Con respecto a la región de procedencia del llamado, la mayor tasa de exposición a agentes de riesgo biológico laboral se observó en las regiones de Los Ríos y Los Lagos (22,7 x 100.000 trabajadores ocupados), seguida por la Región de Aisén $\left(13,3^{\star} 100.000\right.$ trabajadores ocupados) y por la Región Metropolitana (1,2 x 100.000 trabajadores ocupados). No se observaron llamados por este tipo de exposición laboral desde las regiones del norte del país (Tabla 8).

Los llamados fueron realizados principalmente desde un recinto hospitalario $(50,6 \%)$ y desde el hogar $(39,0 \%)$ (Tabla 9).

En relación al interlocutor, en primer lugar 
Tabla 3. Distribución de exposiciones laborales a vacunas y medicamentos inyectables de uso veterinario

\begin{tabular}{|c|c|c|c|c|}
\hline Vacuna & Tipo & Forma & $\%$ & (n) \\
\hline Antirickettsia salmones & $\begin{array}{l}\text { Cultivos de virus de necrosis pancreática infecciosa, } \\
\text { Piscirickettsia salmonis }\end{array}$ & Inactivada & 50,0 & 16 \\
\hline Salmonella enteritidis aves & Antígeno de Salmonella enteritidis fago tipo 4 & Inactivada & 6,3 & 2 \\
\hline Clostridium bovino & $\begin{array}{l}\text { Bacterina-toxoide compuesta por bacterias muertas de } \\
\text { Clostridium chauvoei, septicum, haemolyticum, novyi, } \\
\text { tetani y perfringens tipos C \& D }\end{array}$ & Inactivada & 6,3 & 2 \\
\hline $\begin{array}{l}\text { Virus ISA (anemia infecciosa } \\
\text { del salmón) }\end{array}$ & $\begin{array}{l}\text { Proteínas recombinantes de virus de la anemia infeccio- } \\
\text { sa del Salmón del género Isavirus (Orthomyxoviridae), } \\
\text { producidas en cultivo de levaduras }\end{array}$ & Inactivada & 3,1 & 1 \\
\hline $\begin{array}{l}\text { Cólera aviar (Pasteurella } \\
\text { multocida) }\end{array}$ & Bacterina Pasteurella multocida tipos $1,3,4$ y $3 \times 4$ & Inactivada & 3,1 & 1 \\
\hline $\begin{array}{l}\text { Neumonía cerdos } \\
\text { (Mycoplasma hyopneumoniae) }\end{array}$ & Cultivo de Mycoplasma hyopneumoniae & Inactivada & 3,1 & 1 \\
\hline Brucelosis bovina & $\begin{array}{l}\text { Cepa mutante rugosa de Brucella abortus cepa } 2308 \\
\text { virulento }\end{array}$ & $\begin{array}{l}\text { Viva, altamente } \\
\text { atenuada }\end{array}$ & 3,1 & 1 \\
\hline Antirrábica gatos & Cultivo de virus Rabia & Inactivada & 3,1 & 1 \\
\hline Influenza y tétanos equino & Subunidades proteicas del virus de la influenza equina & Inactivada & 3,1 & 1 \\
\hline Tuberculina PPD bovina (TBC) & $\begin{array}{l}\text { Derivado proteico purificado de Mycobacterium bovis. } \\
\text { Diagnóstico de la tuberculosis bovina }\end{array}$ & Inactivada & 3,1 & 1 \\
\hline Veterinaria no especificada & & - & 6,3 & 2 \\
\hline Solución para eutanasia T61 & No es vacuna* & - & 9,4 & 3 \\
\hline Total & & & 100,0 & 32 \\
\hline
\end{tabular}

*A pesar de no ser vacuna, se consideró exposición de riesgo biológico ocupacional debido a que el trabajador sufrió el accidente luego de utilizar la aguja en un animal.

Tabla 4. Distribución de exposiciones laborales a agentes de riesgo biológico ocupacional según vía de exposición

\begin{tabular}{|lrr|}
\hline Vía de exposición & \% & (n) \\
$\begin{array}{l}\text { Parenteral: inyecciones/lesiones corto } \\
\text { punzantes }\end{array}$ & 39,0 & $(30)$ \\
Picaduras/mordeduras & 37,7 & $(29)$ \\
Digestiva & 9,0 & $(7)$ \\
Cutánea & 6,5 & $(5)$ \\
Ocular & 5,2 & $(4)$ \\
Inhalatoria & 2,6 & $(2)$ \\
Total & 100,0 & $(77)$ \\
\hline
\end{tabular}

se ubica el médico tratante o personal de la salud $(58,4 \%)$, en segundo lugar el propio trabajador expuesto $(22,1 \%)$ y posteriormente un familiar $(14,3 \%)$ (Tabla 10).

Con respecto a la distribución según estación del año, las exposiciones se produjeron principalmente durante las estaciones de verano $(37,7 \%)$ y primavera $(22,0 \%)$ (Tabla 11$)$.

\section{Discusión}

A pesar de que las exposiciones a agentes de riesgo biológico representan sólo 4,3\% del total de exposiciones a tóxicos laborales registradas durante el mismo período, constituyen un grupo 
Exposición a agentes de riesgo biológico en trabajadores chilenos - P. Cerda et al

Tabla 5. Distribución de exposiciones laborales a agente de riesgo biológico ocupacional según familia de agente y sexo

\begin{tabular}{|lccrrrr|}
\hline Agente & \multicolumn{2}{c}{ Masculino } & \multicolumn{2}{c}{ Femenino } & \multicolumn{2}{c|}{ Total } \\
& \% & (n) & \% & (n) & \% & (n) \\
Alimento/bebida contaminados & 3,0 & $(1)$ & 4,5 & $(2)$ & 3,9 & $(3)$ \\
Animales, arañas, insectos & 24,2 & $(8)$ & 59,1 & $(26)$ & 44,1 & $(34)$ \\
Vacuna/medicamento uso veterinario & 66,7 & $(22)$ & 31,8 & $(14)$ & 46,8 & $(36)$ \\
Plantas, hongos, algas & 6,1 & $(2)$ & 4,5 & $(2)$ & 5,2 & $(4)$ \\
Total & 100,0 & $(33)$ & 100,0 & $(44)$ & 100,0 & $(77)$ \\
\hline
\end{tabular}

Tabla 6. Riesgo de exposición laboral a mordedura de araña, animales y picadura de insecto asociado a sexo femenino

\begin{tabular}{|c|c|c|c|c|c|c|}
\hline Sexo & $\begin{array}{c}\text { Arañas, animales } \\
\text { e insectos } \\
\text { (n) }\end{array}$ & $\begin{array}{l}\text { Otros agentes de } \\
\text { riesgo biológico } \\
\text { (n) }\end{array}$ & $\begin{array}{l}\text { Total } \\
\text { (n) }\end{array}$ & OR & $I_{95 \%}$ & Valor $p$ \\
\hline Femenino & $26(33,8 \%)$ & $18(23,3 \%)$ & $44 \quad(57,1 \%)$ & 4,5 & $1,5-13,9$ & $<0,005$ \\
\hline Masculino & $8(10,4 \%)$ & $25(32,5 \%)$ & $33 \quad(42,9 \%)$ & & & \\
\hline Total & $34(44,2 \%)$ & $43(55,8 \%)$ & 77 (100\%) & & & \\
\hline
\end{tabular}

$\chi^{2}=9,2$

Tabla 7. Riesgo de exposición laboral a vacunas veterinarias y medicamentos de uso veterinario asociado a sexo masculino

\begin{tabular}{|c|c|c|c|c|c|c|c|}
\hline Sexo & $\begin{array}{l}\text { Vacuna veterinaria, } \\
\text { medicamento veterinario } \\
\text { (n) }\end{array}$ & $\begin{array}{l}\text { Otros agentes de } \\
\text { riesgo biológico } \\
\text { (n) }\end{array}$ & & $\begin{array}{l}\text { Total } \\
\text { (n) }\end{array}$ & OR & IC 95\% & Valor $\mathbf{p}$ \\
\hline Masculino & $22(28,6 \%)$ & $11(14,3 \%)$ & 33 & $(42,9 \%)$ & 4,2 & $1,4-12,6$ & $<0,005$ \\
\hline Femenino & $14(18,2)$ & $30(38,9 \%)$ & 44 & $(57,1 \%)$ & & & \\
\hline Total & $36(46,8 \%)$ & $41 \quad(53,2 \%)$ & 77 & $(100 \%)$ & & & \\
\hline
\end{tabular}

$\chi^{2}=9,2$

relevante debido a las consecuencias que podrían generar sobre el estado de salud de los trabajadores afectados.

Una de nuestras principales limitaciones consiste en que la notificación a CITUC es voluntaria, por lo tanto, se sospecha que habría un número mucho mayor de exposiciones a agentes de riesgo biológico a nivel laboral. A pesar de esta limitante, los resultados de este estudio contribuyen a aumentar la información sobre exposición laboral a tóxicos en el país.

El principal agente de riesgo biológico laboral correspondió a vacunas de uso veterinario. Las vacunas veterinarias en su mayoría se utilizan de manera inactivada, sin embargo, en el caso de la vacuna contra brucellosis bovina, cuyo principal riesgo es abortivo, se utiliza viva, aunque altamente atenuada. Según la OMS, la exposición ocupacional a brucellosis puede ocurrir, entre otras vías, a través de la auto-inoculación accidental con vacunas vivas ${ }^{17}$. En este estudio se identificó un caso de inoculación accidental de esta vacuna en un trabajador de sexo masculino.

Además del riesgo biológico asociado al agente, algunos estudios han descrito que la autoinoculación accidental de vacunas con compo- 
Exposición a agentes de riesgo biológico en trabajadores chilenos - P. Cerda et al

Tabla 8. Distribución de exposiciones laborales a agentes de riesgo biológico según región y cálculo de tasa de exposición por trabajadores ocupados

\begin{tabular}{|lccc|}
\hline & $\begin{array}{c}\text { Exposiciones a agente de } \\
\text { riesgo biológico, registro } \\
\text { CITUC (n de trabajadores) }\end{array}$ & $\begin{array}{c}\text { Total trabajadores } \\
\text { ocupados } \\
\text { (INE, 2011)* }\end{array}$ & $\begin{array}{c}\text { Tasa *100.000 } \\
\text { trabajadores } \\
\text { ocupados }\end{array}$ \\
\hline Total nacional & 77 & 7.589 .410 & 1,01 \\
Arica y Parinacota & 0 & 76.140 & 0 \\
\hline Tarapacá & 0 & 150.540 & 0 \\
\hline Antofagasta & 0 & 260.400 & 0 \\
Atacama & 0 & 124.280 & 0 \\
Coquimbo & 0 & 319,14 & 0 \\
\hline Valparaíso & 4 & 778.350 & 0,5 \\
Metropolitana & 38 & 3.147 .180 & 1,2 \\
\hline Libertador B. O'Higgins & 2 & 396.160 & 0,5 \\
Maule & 3 & 441.940 & 0,6 \\
\hline Bio Bío & 3 & 841.200 & 0,3 \\
\hline La Araucanía & 6 & 401.700 & 1,4 \\
\hline Los Ríos y Los Lagos & 12 & 52.630 & 22,7 \\
\hline Aisén Gral. Carlos Ibáñez del Campo & 7 & 52.820 & 13,3 \\
\hline Magallanes y Antártica Chilena & 2 & 73.270 & 2,7 \\
\hline
\end{tabular}

*INE. Trimestre noviembre-diciembre-enero (NDE, 2011).

Tabla 9. Distribución de exposiciones laborales a agente de riesgo biológico según lugar de procedencia del Ilamado

\begin{tabular}{|lrr|}
\hline Lugar de procedencia del llamado & \% & (n) \\
Hospital & 50,6 & $(39)$ \\
Hogar & 39,0 & $(30)$ \\
Servicio de atención primaria & 3,9 & $(3)$ \\
Lugar de trabajo & 3,9 & $(3)$ \\
Clínica veterinaria & 1,3 & $(1)$ \\
Consulta externa & 1,3 & $(1)$ \\
Total & 100,0 & $(77)$ \\
\hline
\end{tabular}

nente oleoso utilizadas en la industria salmonera ha causado grave necrosis de tejidos y espasmos vasculares $^{18}$ y que pinchazos accidentales en el ambiente laboral con espinas de pescado han producido infecciones ${ }^{19}$ que han llegado a requerir amputación de dedos ${ }^{20}$.

Algunos agentes biológicos tienen, además,
Tabla 10. Distribución de exposiciones laborales a agente de riesgo biológico según interlocutor

\begin{tabular}{|lcr|}
\hline Interlocutor & \% & (n) \\
\hline Médico o personal de salud & 58,4 & $(45)$ \\
Trabajador & 22,1 & $(17)$ \\
\hline Familiar & 14,3 & $(11)$ \\
Veterinario & 2,6 & $(2)$ \\
Compañero de trabajo & 2,6 & $(2)$ \\
Total & 100,0 & $(77)$ \\
\hline
\end{tabular}

Tabla 11. Distribución de exposiciones laborales a agente de riesgo biológico según estación

\begin{tabular}{|lcc|}
\hline Estación & \% & (n) \\
\hline Verano & 37,7 & $(29)$ \\
Primavera & 22,0 & $(17)$ \\
Otoño & 20,8 & $(16)$ \\
Invierno & 19,5 & $(15)$ \\
Total & 100,0 & $(77)$ \\
\hline
\end{tabular}


Exposición a agentes de riesgo biológico en trabajadores chilenos - P. Cerda et al

potencial sensibilizante que debe tenerse en cuenta en la evaluación de riesgos en el lugar de trabajo ${ }^{8}$.

El grupo de mayor riesgo de exposición a agentes de riesgo biológico correspondió a trabajadores jóvenes en edad reproductiva. Esta población es especialmente vulnerable al efecto de los tóxicos ${ }^{2}$ y debe ser protegida.

Este estudio identifica a las regiones de Los Ríos, Los Lagos y Aisén como regiones de mayor riesgo de exposición ocupacional a agentes de riesgo biológico. La mayoría de las exposiciones se produjo por contacto con vacunas de uso veterinario, en especial vacunas utilizadas en la industria salmonera, actividad que se desarrolla principalmente en estas regiones ${ }^{21}$.

Otro resultado destacado en relación a agente correspondió a mordeduras de araña de rincón, ubicándose en segundo orden de importancia. La mordedura por arácnidos ponzoñosos es, afortunadamente, reconocida en Chile como un accidente laboral y todo trabajador que es mordido en su puesto de trabajo debe recibir atención médica, hospitalización, medicamentos ${ }^{22}$. Según los resultados, las mujeres son quienes se encuentran en mayor riesgo de sufrir mordedura por este arácnido en el lugar de trabajo, probablemente porque son quienes desarrollan con mayor frecuencia actividades de limpieza. Este resultado es similar al observado previamente en población general ${ }^{23}$.

Llama la atención que no se recibieron llamados por exposición a agentes de riesgo biológico ocupacional en profesionales o trabajadores de la salud, a pesar de que en Chile las exposiciones de riesgo biológico en personal de la salud son frecuentes ${ }^{24}$, probablemente porque cuando se produce este accidente laboral, el trabajador afectado tiene una instancia donde notificarlo y solicitar asesoría en su respectivo servicio asistencial. Además existen normas ministeriales en relación a esta materia ${ }^{25-26}$ y la consulta a CITUC puede resultar innecesaria.

Para prevenir intoxicaciones de origen laboral es fundamental identificar a los trabajadores en riesgo y realizar más estudios, que involucren a un mayor número de trabajadores. Factores de riesgo de intoxicación laboral relacionados con desconocimiento del efecto sobre la salud de agentes de riesgo biológico, el uso de equipos de protección y la susceptibilidad individual no fueron investigados en este estudio y podrían ser objeto de futuras investigaciones. Estudios han determinado que la actitud del trabajador y su comportamiento, influido por su propia percepción del riesgo, podría en gran medida subestimar el riesgo real ${ }^{7}$ y que el grado de conocimiento y percepción del riesgo en trabajadores ocupacionalmente expuestos a riesgos biológicos varía según la ocupación ${ }^{4}$.

En el ámbito de políticas públicas, se sugiere la notificación obligatoria de toda intoxicación de origen laboral. Este riesgo emergente podría requerir vigilancia activa por sus potenciales consecuencias en salud pública.

\section{Referencias}

1. Albrecht A, Kiel K, Kolk A. Strategies and methods for investigation of airborne biological agents from work environments in Germany. Int J Occup Saf Ergon 2007; 13: 201-13.

2. OIT (Organización Internacional del Trabajo) 2010. Riesgos emergentes y nuevos modelos de prevención en un mundo de trabajo en transformación. Disponible en: http://www.ilo.org/wcmsp5/groups/public/---ed_ protect/---protrav/-- safework/documents/publication/ wcms_124341.pdf

3. Haagsma JA, Tariq L, Heederik DJ, Havelaar AH. Infectious disease risks associated with occupational exposure: a systematic review of the literature. Occup Environ Med 2012; 69 (2): 140-6.

4. De Giusti M, Corrao CR, Mannocci A, Palazzo C, Riccardi R, Schmidt SL, Sernia S, La Torre G. Occupational biological risk knowledge and perception: results from a large survey in Rome, Italy. Ann Ist Super Sanita 2012; 48 (2): 138-45.

5. Vives A, Jaramillo J. Salud laboral en Chile. Arch Prev Riesgos Labor 2010; 13 (3): 150-6.

6. MINSAL (Ministerio de Salud de Chile), 2007. Norma técnica de vigilancia de intoxicaciones agudas por plaguicidas REVEP. Disponible en http://epi.minsal.cl/epi/ html/normas/normaREVEP.pdf

7. Corrao CR, Mazzotta A, LA Torre G, DE Giusti M. Biological risk and occupational health. Ind Health 2012; 50 (4): 326-37.

8. OSHA (Occupational Safety and Health Administration) 2007. Riesgos laborales derivados de agentes biológicos: retos que hay que afrontar. Disponible en: http://osha. europa.eu/es/seminars/occupational-risks-from-biological-agents-facing-up-the-challenges-es

9. Cediel B, Natalia M, Villamil LC. Riesgo biológico ocupacional en la medicina veterinaria, área de intervención prioritaria. Rev Salud Pública 2004; 6 (1): 28-43. 
10. Berkelman RL. Human illness associated with use of veterinary vaccines. Clin Infect Dis 37 2003; 407-14.

11. Ministerio de Salud de Chile (MINSAL), Dirección del Trabajo (DT) e Instituto de Seguridad Laboral (ISL). ENETS 2009-2010 "Primera Encuesta Nacional de Condiciones de Empleo, Trabajo Calidad de Vida y Salud de los Trabajadores y Trabajadoras en Chile". Disponible en: http://www.eligevivirsano.cl/wp-content/ uploads/2012/01/Informe-Encuesta-ENETS-2009-2010. pdf

12. INE 2011. Población total de 15 años y más por situación en la fuerza de trabajo, nivel nacional y regional según sexo. Disponible en http://www.ine.cl/canales/ chile_estadistico/mercado_del_trabajo/nene/cifras_trimestrales_NDE_2011.php

13. Communication Record. Disponible en http://www. who.int/ipcs/poisons/intox_dms/en/ (Spanish format of the communication record).

14. WHO. International Programme on Chemical Safety. Disponible en http://www.who.int/ipcs/en/

15. Dutkiewicz J, Jabłoński L, Olenchock SA. Occupational biohazards: a review. Am J Ind Med 1988; 14 (5): 60523.

16. Dutkiewicz J, Cisak E, Sroka J, Wójcik-Fatla A, Zając V. Biological agents as occupational hazards - selected issues. Ann Agric Environ Med 2011; 18 (2): 286-93.

17. WHO (World Health Organization) 2006. Brucellosis in human and animals. www.who.int/csr/resources/
publications/Brucellosis.pdf

18. Leira HL, Baalsrud KJ. Operator safety during injection vaccination of fish. Dev Biol Stand 1997; 90: 383-7.

19. Myers ML. Review of occupational hazards associated with aquaculture. J Agromedicine 2010; 15 (4): 412-26.

20. Charmish B. Vibrio hits Israel; tilapia sales. Fish Farmer 1996; 10 (5): 17.

21. Bravo S, Midtlyng PJ. The use of vaccines in the Chilean salmon industry 1999-2003 Aquaculture 2007; 270: 36 42.

22. ISP (Instituto de Salud Pública de Chile) 2005. Riesgo Laboral por mordedura de araña de los rincones. Disponible en: http://www.ispch.cl/encabezado/noticias/ publica/boletinfinal.pdf

23. Ríos J, Pérez M, Sánchez M, Bettini M, Mieres J, Paris E. Caracterización clínico-epidemiológica telefónica de la mordedura por araña de rincón, en un centro de información toxicológica de Chile, durante el año 2005. Rev Med Chile 2007; 135: 1160-5.

24. Villarroel J, Bustamante MC, Manríquez I, Bertoglia MP, Mora M, Galarce N. Exposición laboral a fluidos corporales de riesgo en el Hospital Clínico Félix Bulnes Cerda durante 11 años de estudio. Rev Chil Infectol 2012; 29 (3): 255-62.

25. Guía Clínica 2010. Manejo y Tratamiento de la Infección por Virus de Hepatitis B. MINSAL.

26. Norma Técnica No 48 Ministerio de Salud. Decreto Exento No 561 del 7 de marzo de 2000. 